\title{
Biomechanical stress analysis of the main soft tissues associated with the development of adult acquired flatfoot deformity
}

\author{
Christian Cifuentes De la Portilla a, c, *, Ricardo Larrainzar-Garijo ${ }^{\text {, }}$ Javier Bayod ${ }^{\mathrm{a}}$ \\ a Applied Mechanics and Bioengineering Group (AMB), Aragón Institute of Engineering Research (I3A), Universidad de Zaragoza, Spain \\ b Orthopaedics and Trauma Department, Surgery Department, Hospital Universitario Infanta Leonor, Madrid, Spain \\ ${ }^{\mathrm{c}}$ Facultad de Ciencias Médicas - Departamento de Medicina, Universidad Espíritu Santo, Ecuador
}

\section{ART ICLE INFO}

\section{Keywords:}

Biomechanics

Flatfoot

FE modeling

Tibialis tendon dysfunction

\begin{abstract}
A B S T R A C T
Background: Adult acquired flatfoot deformity (AAFD) is traditionally related to a tibialis posterior tendon deficiency. In the intermediate stages, treatments are commonly focused on reinforcing this tissue, but sometimes the deformation appears again over time, necessitating the use of more aggressive options. Tissue stress cannot be consistently evaluated through traditional experimental trials. Computational foot modeling extends knowledge of the disease and could help guide the clinical decisions. This study analyzes the biomechanical stress of the main tissues related to AAFD and their capacity to support the plantar arch.

Methods: A FE foot model was reconstructed. All the bones, cartilages and tissues related to AAFD were included, respecting their biomechanical characteristics. The biomechanical tissue stress was quantified. The capacity of each soft tissue to support the plantar arch was measured, following clinical criteria.

Findings: Biomechanical stress of the tibialis posterior tendon is considerably superior to both the plantar fascia and spring ligament stress. However, it cannot maintain the plantar arch by itself. Both the tibialis posterior tendon and spring ligament act in reducing the hindfoot pronation, while the plantar fascia is the main tissue that prevents arch elongation. The Achilles tendon action increases the plantar tissue stress.

Interpretation: The tibialis posterior tendon stress increases when the spring ligament or the fascia plantar fails. These findings are consistent with the theory that regards the tibialis posterior tendon as a secondary actor because it cannot support the plantar arch and claudicates when the hindfoot has rotated around the talonavicular joint.
\end{abstract}

\section{Introduction}

Adult acquired flatfoot deformity (AAFD) is a pathology that causes a progressive flattening of the foot arch, traditionally related to the weakening of the Tibialis Posterior Tendon (TPT) (Smyth et al., 2017). Many treatment options are focused on reinforcing this tissue, but sometimes the foot deformation appears again over time, necessitating the use of more aggressive and restrictive options such as selective arthrodesis in the middle and hindfoot joints or corrective osteotomies of the hindfoot valgus moment (Fowble and Sands, 2004; Guha and Perera, 2012; Lladó et al., 2015; Toullec, 2015; Bluman et al., 2007; Vulcano et al., 2013; Valladar et al., 2015). This scenario often appears in the intermediate stages of the disease and currently there is no accepted consensus on the appropriate treatment (Fowble and Sands,
2004). Some clinical studies have found that this deformity is also related with the failure of other soft tissues, such as the plantar fascia (PF) or the plantar calcaneonavicular ligament, also known as the spring ligament (SL) (Lee et al., 2005; Shibuya et al., 2010; Toullec, 2015).

Furthermore, some biomechanical variables such as soft tissue biomechanical stress have not been adequately evaluated through experimental trials. The main limitations of such trials are the considerable biomechanical complexity of the foot, the difficulty of tissue segmentation during experimental tests and other limitations related to measuring the whole tissue during loading tests (Huang et al., 1993; Song et al., 1996; Rabbito et al., 2011). Many researchers have analyzed normal and pathological human foot biomechanics using different modeling approaches (Huang et al., 1993; Bertani et al., 1999; Deland, 2001; Cheung et al., 2005; Vulcano et al., 2013). Some studies use cadaver

\footnotetext{
* Corresponding author.

Email address: cjcifuentesd@unal.edu.co (C.C. De la Portilla)
} 
models to evaluate the reaction of the foot under progressive loads. This allows the measurement of the anatomical deformation of both the soft tissues and bone structure of the foot, generated by the loading tests (Pichler et al., 2005; Deschamps et al., 2011; Marchena et al., 2013; Toullec, 2015). However, these kinds of studies require high economic investments in measurement equipment, as well as meticulous control over the tested tissues to guarantee their biomechanical characteristics. Thus, it is extremely difficult to obtain isolated information about each tissue with consistent results (Morales Orcajo et al., 2015).

An alternative approach accepted nowadays by clinicians and biomechanical engineers is the finite elements (FE) modeling. Computational foot modeling extends knowledge of AAFD and could help guide clinical decisions (Smith, 2015). This computational methodology allows the design of complex numerical models that adequately represent human foot behavior for biomechanics studies (Wang et al., 2016a). These models are a valid alternative that allow tissue alterations or loading changes to be included in virtual structures that cannot easily be considered when real tissues are used (Wang et al., 2015; Morales Orcajo et al., 2015; Smith, 2015; Smyth et al., 2017; Wong et al., 2017). Of course, their validity requires the proper design of the physiological structures and the adequate modeling of the mechanical properties of the tissue (Viceconti et al., 2005). These models use tissue model parameters taken from experimental cadaver foot studies, generally simplifying the shape or behavior to optimize the model calculation. As mentioned above, clinical studies have identified some soft tissues that have an important involvement in AAFD development: the TPT (Lee et al., 2005; Toullec, 2015), PF (Haddad et al., 2011; Hidalgo et al., 2014) and the SL. However, currently published models simulate these tissues but without considering their geometry appropriately (Wang et al., 2015; Smith, 2015; Smith et al., 2016; Wang et al., 2016b). They mostly consider these tissues as bar elements (unidimensional simple elements) which generate an adequate foot deformation but does not allow relevant biomechanical aspects to be measured such as the tissue traction stress. This limits the clinical information that these models can provide. Accurate mechanical measurements are important for the analysis of AADF development and its effect on foot tissues (Smyth et al., 2017).

The main objective of this study was to evaluate the biomechanical stress generated on the tibialis posterior tendon, the plantar fascia and the spring ligament in different scenarios, in order to analyze their capacity to maintain the plantar arch, to quantify the foot deformation generated, and to identify how these tissues failure could generate a flatfoot deformity. The proposed FE model proposed, considers both the geometry and biomechanical characteristics of the human foot tissue. Common clinical metrics used in AAFD diagnosis were used to evaluate the talocalcaneal divergence and both the fall and lengthening of the plantar arch (the main signs of the pathology). These are the internal Moreau-Costa-Bertani (IMCB) angle, the forefoot abduction (FA) angle and the Kite's angle.

\section{Methods}

\subsection{FE model design}

This study is based on the model proposed by Morales Orcajo et al. (2015), developed by our research group. This model reconstructs a healthy human unloaded foot, based on CT- images acquired from the right foot of a 49-year-old male - weight: $720 \mathrm{~N}$ and height: $1.70 \mathrm{~m}$. Segmentation and tissue reconstruction were performed using MIMICS V. 10 software (Materialize, Leuven, Belgium). The model maintains the morphology of the cartilage, tendons and the plantar fascia as well as the differentiation of the cortical and trabecular bone (Fig. 1-A). This bone representation is generally overlooked, despite the importance of considering this differentiation in bone mechanics modeling, mainly in tissue stress evaluations (Garcia-Aznar et al., 2009; Bayod et al., 2012). Due to the difficulty of segmenting the SL and both the short plantar ligament (SPL) and long plantar ligament (LPL) from the CT-images, the geometry of these tissues was included using atlas images, following the surgeons' guidance (See Fig. 1-B). The SPL and the LPL have a secondary role in plantar arch maintenance (Tao et al., 2010), but these tissues are fundamental for an adequate analysis of AAFD development (Hidalgo et al., 2014; Steginsky and Vora, 2017).

\subsubsection{Meshing}

The model meshing was performed using ANSYS V.15 (Canonsburg, Pennsylvania, United States). A trial-error approach was employed to optimize the mesh size of each segment (Burkhart et al., 2013). The conditions to achieve a reasonable mesh size without compromising the calculation time were:

- A minimum mesh size sufficiently small to fit into the tightest segments, particularly in the forefoot where many different minor components are concentrated.

- A maximum mesh size consistent with the minimum to avoid large differences in element size between regions. This was performed to ensure that the results were independent of the mesh density.

- A mesh accuracy in the $99 \%$ of the elements better than 0.2 (mesh quality - Jacobians) and check that the poor elements were located away from the region of highest interest.

- The number of elements below 500,000 with our current computational capacity (Intel Core i5 $3.2 \mathrm{GHz}$ CPU and $8 \mathrm{~GB}$ RAM), meshes higher than one hundred thousand elements increase significantly the computational time.

The equilibrium among these goals was found with 256,598 linear tetrahedral elements (C3D4) with element sizes as follows: $1 \mathrm{~mm}$ for the smallest cartilages between phalanges, $2 \mathrm{~mm}$ for phalanges, the thinnest ligaments and the rest of the cartilages, $3 \mathrm{~mm}$ for metatarsals and the rest of the tendons, and $5 \mathrm{~mm}$ for the large bones in the hindfoot. The mesh quality was checked using the recommendations of Burkhart et al. (2013) as a reference. They suggest that to obtain reliable results, the percentage of the inaccurate elements for each one of the criteria shown in Table 1 must be less than 5\%. All parameters were within good mesh quality ratios (see Table 1). All the simulations were conducted with Abaqus/CAE 6.14-1 (Dassault Systémes, Vélizy-Villacoublay, France) using the Nonlinear geometry solver.

\subsection{Tissue modeling and contour conditions}

The model includes the geometry of all the main soft tissues related to both AAFD development and the foot loading response, in appropriate anatomical positions as shown in (Fig. 1-B).

Except in the case of the cartilage model, the tissue models were considered as elastic-linear elements using biomechanical properties reported in the literature: Cortical bone $(E=17000 \mathrm{MPa}, v=0.3)$ (Morales Orcajo et al., 2015), trabecular bone ( $E=700 M P a, v=0.3)$ (Morales Orcajo et al., 2015), tendons $(E=450 M P a, v=0.3)$ (Garcia-Aznar et al., 2009), ligaments $(E=250 M P a, v=0.28$ ) (Tao et al., 2010; Morales Orcajo et al., 2015), and PF ( $E=240 M P a, v=0.28$ ) (Wright and Rennels, 1964; Morales Orcajo et al., 2015), " $E$ ” being the Young's modulus and " $v$ " the Poisson's ratio. Some other models consider cartilage tissue as an elastic-linear element (Tao et al., 2010). However, in our case this assumption increased the rigidity of our model, reducing the bones rotation and the foot structure deformation, both of which are required to obtain the signs of AAFD: hindfoot pronation and forefoot abduction. To improve the model behavior and its convergence, we model the cartilage material as non-linear and hyperelastic, as suggested by Mansour and Wu (Mansour, 2003; Wu, 


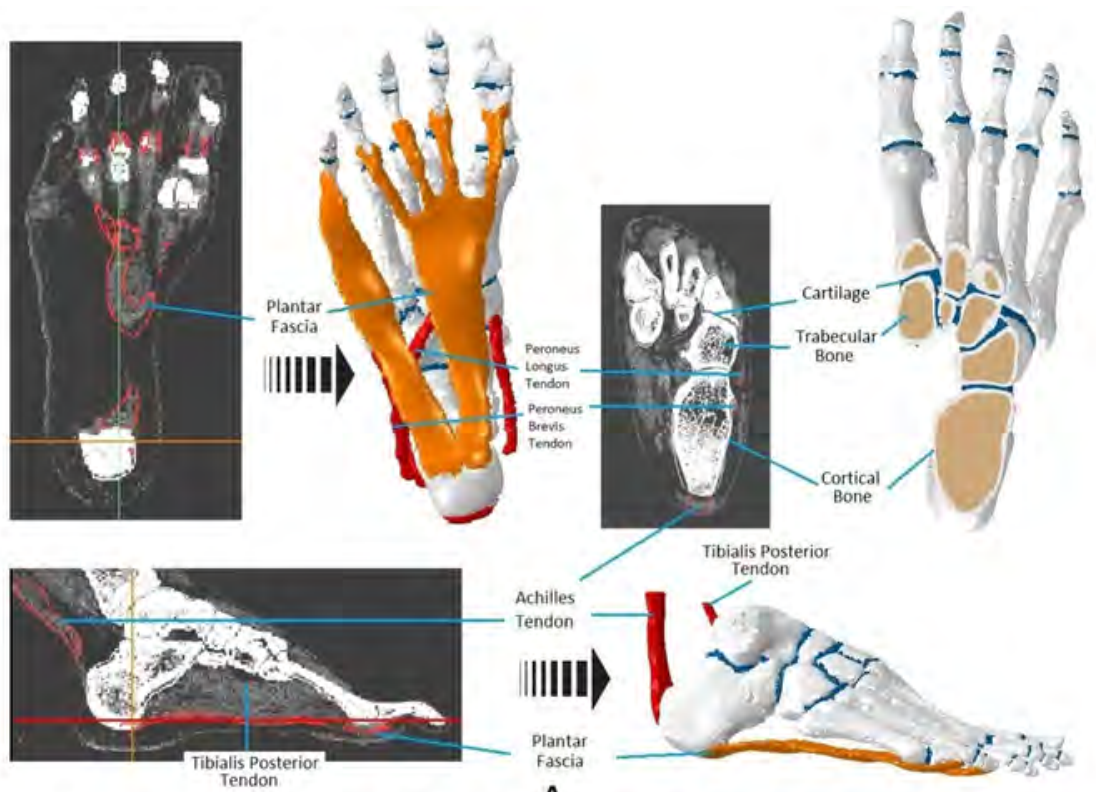

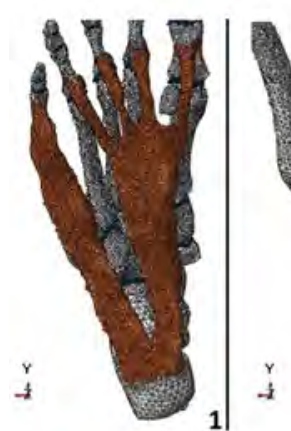
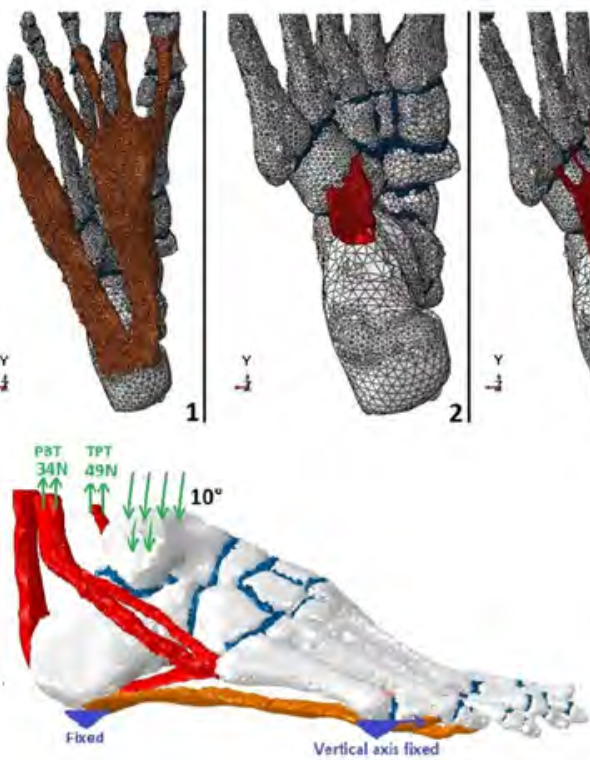

A

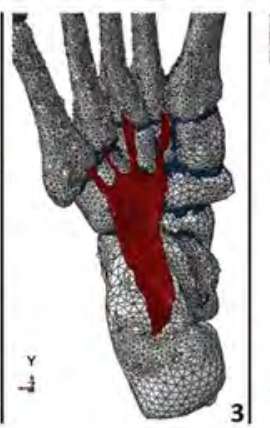

B
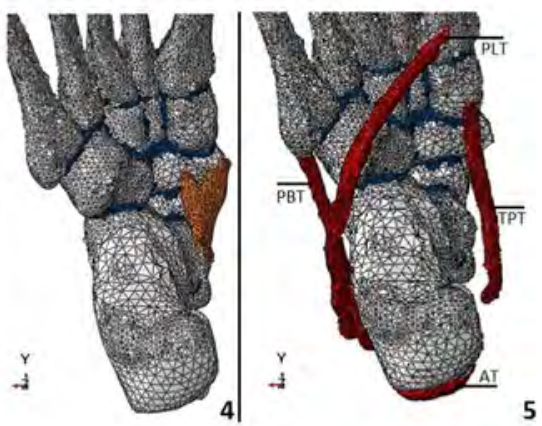

5

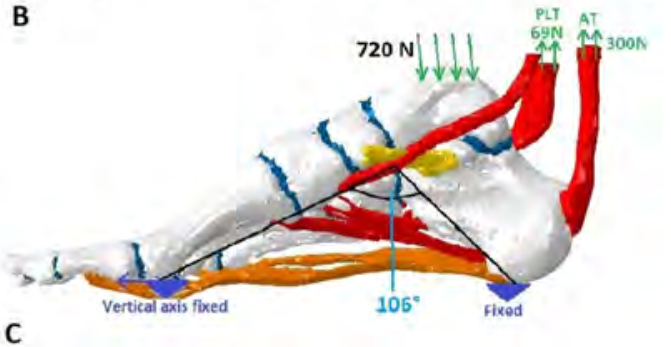

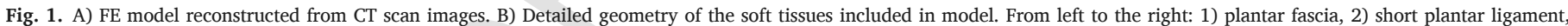

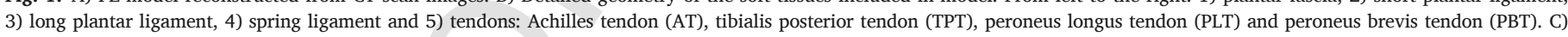
Loading settings applied to the model, including the boundary conditions and the traction forces applied to the tendons.

Table 1

Mesh quality metrics based on Burkhart et al. (2013) recommendations.

\begin{tabular}{|c|c|c|c|}
\hline Quality metric & $\begin{array}{l}\text { (Assessment } \\
\text { criteria) }\end{array}$ & $\begin{array}{l}\text { Accurate } \\
\text { elements }\end{array}$ & $\begin{array}{l}\text { Inaccurate } \\
\text { elements }\end{array}$ \\
\hline $\begin{array}{l}\text { Element } \\
\text { Jacobians }\end{array}$ & $>0.2$ & $99.7 \%$ & $0.3 \%$ \\
\hline Aspect ratio & $<3$ & $94.5 \%$ & $4.5 \%$ \\
\hline Min. angles & $>30^{\circ}$ & $97.2 \%$ & $2.8 \%$ \\
\hline Max. angles & $>120^{\circ}$ & $98.8 \%$ & $1.2 \%$ \\
\hline
\end{tabular}

2007), using the Ogden model. Additionally, these studies demonstrate that the cartilage tissue displaces water under compression. Therefore, it is not entirely correct to consider it as a quasi-incompressible material as is generally considered (Tao et al., 2010; Morales Orcajo et al., 2015). Its strain energy density function $U$ is:
$U=\frac{\mu}{\alpha^{2}}\left(\lambda_{1}{ }^{\alpha}+\lambda_{2}{ }^{\alpha}+\lambda_{3}{ }^{\alpha}-3\right)+\frac{1}{D}(J-1)^{2}$

where $\mu$ is the initial shear modulus, $\alpha$ is the strain hardening exponent, and $D$ is the compressibility parameter. The parameters used are: $\mu=4.4, \alpha=2$ and $D=0.45$.

Considering that the CT- images used in the model reconstruction were taken from an unloaded patient foot, an initial simulation was performed to obtain a normal standing load position (midstance of the gait cycle). This case was simulated including all the soft tissues considered in our model, applying a load of $720 \mathrm{~N}$ which represents the full weight of an adult person leaning on one foot, with which the traditional scenario of the AAFD diagnostic assessment was emulated. This load was introduced in descending vertical direction and $10^{\circ}$ of inclination (distributed in the zone of contact Tibia - Astragalus (90\%) and Fibula - Astragalus (10\%)) as Morales Orcajo et al. (2015) suggest. The 
traction force of the tendons was included as is reported in the Arangio and Salathe (2009) study. The simulations were performed maintaining fixed nodes at the lower part of the calcaneus and blocking the Z-axis displacement (vertical) of the lower nodes of the first and fifth metatarsals. In this way the ground effect over the model at the midstance of the gait cycle was simulated (See Fig. 1-C)

Table 2

Summary of the simulations and their description. The bones and cartilages were included in all the simulations.

\begin{tabular}{|c|c|}
\hline Simulation & Description \\
\hline Normal & $\begin{array}{l}\text { Normal standing load simulation, including all the soft } \\
\text { tissues }\end{array}$ \\
\hline Max. Deformation & $\begin{array}{l}\text { Simulation performed keeping only bones and } \\
\text { cartilages }\end{array}$ \\
\hline Passive tissues evaluation & Simulation performed removing all the tendons \\
\hline $\begin{array}{l}\text { TPT evaluation - Scenario } \\
\quad 1\end{array}$ & Maintaining only the tendons: TPT, AT, PBT and PLT \\
\hline $\begin{array}{l}\text { TPT evaluation - Scenario } \\
\quad 2\end{array}$ & $\begin{array}{l}\text { Keeping only the TPT, removing all the other soft } \\
\text { tissues }\end{array}$ \\
\hline $\begin{array}{l}\text { TPT evaluation - Scenario } \\
3\end{array}$ & Maintaining only both the TPT and the PF \\
\hline $\begin{array}{l}\text { TPT evaluation - Scenario } \\
4\end{array}$ & Maintaining only both the TPT and the SL \\
\hline $\begin{array}{l}\text { TPT evaluation - Scenario } \\
5\end{array}$ & Maintaining only both the TPT and the AT \\
\hline $\begin{array}{l}\text { PF evaluation - Scenario } \\
\quad 1\end{array}$ & Maintaining all the tendons and the PF \\
\hline $\begin{array}{l}\text { PF evaluation - Scenario } \\
\quad 2\end{array}$ & Keeping only the PF \\
\hline $\begin{array}{l}\text { PF evaluation - Scenario } \\
3\end{array}$ & Maintaining only the PF, TPT and AT \\
\hline $\begin{array}{l}\text { PF evaluation - Scenario } \\
\quad 4\end{array}$ & Maintaining only both the PF and TPT \\
\hline $\begin{array}{l}\text { SL evaluation - Scenario } \\
\quad 1\end{array}$ & Maintaining all the tendons and the SL \\
\hline $\begin{array}{l}\text { SL evaluation - Scenario } \\
\quad 2\end{array}$ & Keeping only the SL \\
\hline $\begin{array}{l}\text { SL evaluation - Scenario } \\
\quad 3\end{array}$ & Maintaining only the SL, TPT and AT \\
\hline $\begin{array}{l}\text { SL evaluation - Scenario } \\
\quad 4\end{array}$ & Maintaining only both the SL and TPT \\
\hline
\end{tabular}

\subsection{Model analysis and evaluation criteria}

The biomechanical tissue stress was quantified using the field output spectrum available in Abaqus. The parameter used in the evaluation process was the maximum principal stress (S.Max). These eigenvalues are closely related to the tensile stress generated in foot tissues during loading. Each of the simulated case was normalized using as a reference the normal standing load simulations results. In order to identify the tissue contribution in both the plantar arch maintenance and hindfoot pronation momentum, different combinations of tissue failure/ weakness were simulated. This strategy allowed us to identify both the tissue stress and the foot structure alteration. All the simulations performed are summarized in Table 2. The effect of both the PLT and PBT were not considered, because their relationship with AAFD development is not a determinant (Toullec, 2015). The structural changes of the plantar arch were quantified by determining the IMCB angle, the talocalcaneal divergence through Kite's angle and the forefoot abduction (FA) angle, measured as shown in Fig. 3. The application Ruler developed by Ergonautas (2017) and the Polytechnic University of Valencia was used for the angles measurements. All the angle units are in degrees.

To determine the maximum deformation values that our model generates and to calculate the relative contribution of each of the analyzed tissues, a simulation eliminating all the soft tissues (except cartilages) was performed (Maximum deformation). This method follows the methodology used by Tao et al. (2010) in a real tissue experimental test.

\subsection{FE foot model validation}

The model was validated following the recommendations of Tao et al. (2009). They measured some anatomical points in two different loading conditions (light loading and normal standing load), from the lateral view of RX-images. The variation of these points allowed us to compare the vertical displacements visible in radiographic images in normal feet with our FE model predictions. The vertical distance of the highest point of the Astragalus (AST), Navicular (NAV), the middle of the Cuneiform (CUN) and the highest point of the first metatarsal head (MTH1) were measured, as can be seen in Fig. 2. These measurements were performed on 12 radiographic images of healthy patients to ob-
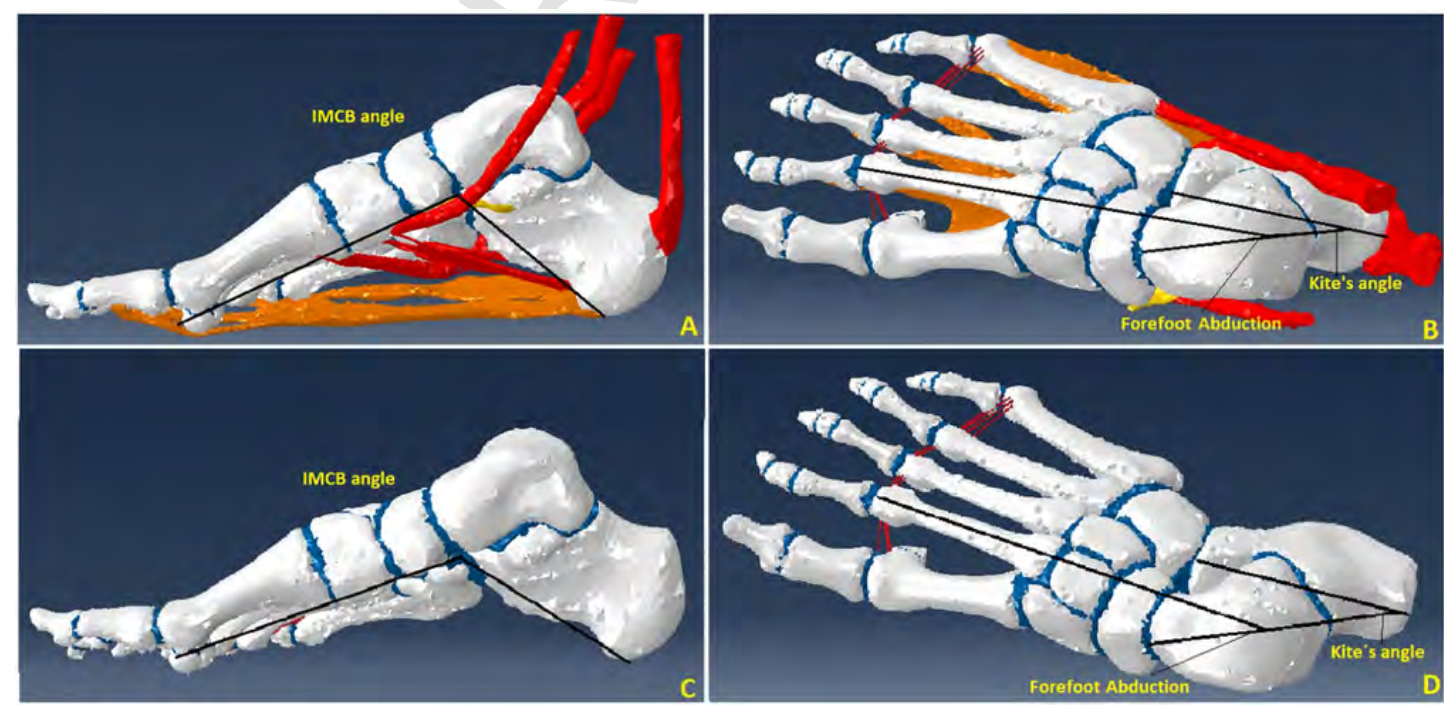

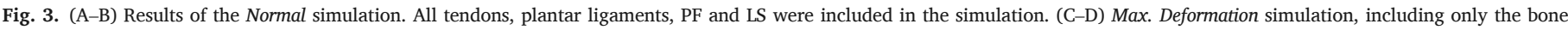
structure and cartilages. 

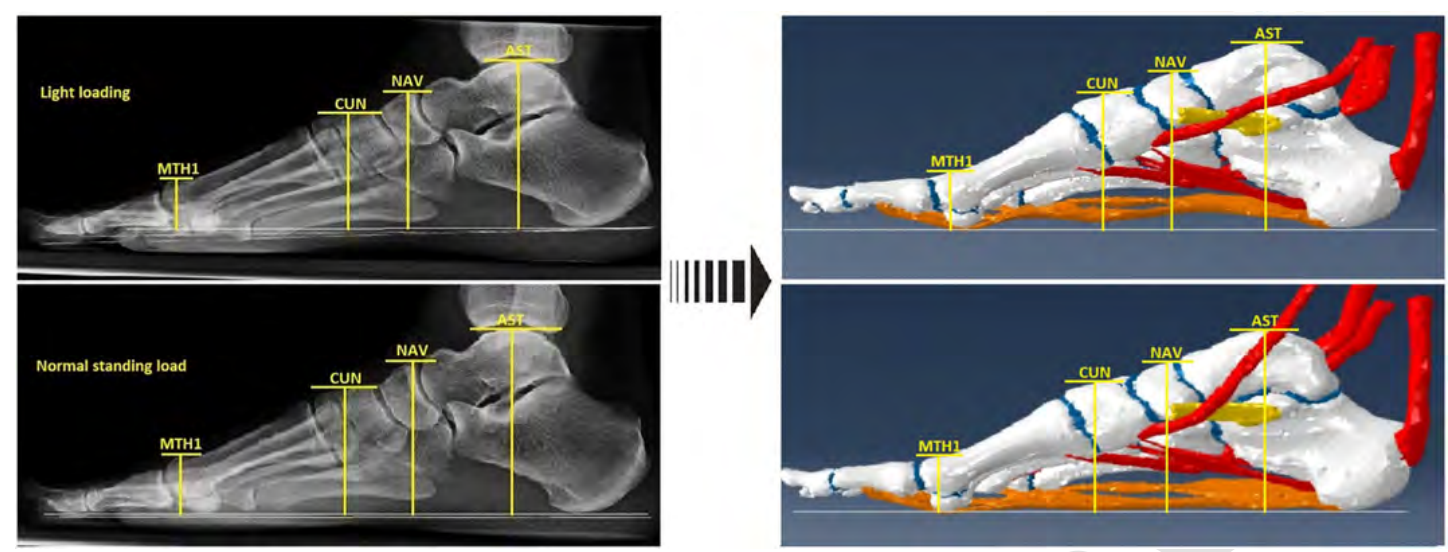

Fig. 2. Model validation using the method proposed by Tao (Tao et al., 2009). The image shows the comparison between a real radiographic image and the model simulation.

tain a normalized average and standard deviation that allow an objective comparison with the model predictions. The free software ImageJ was used to measure the distances. Each image (96 ppi (pixels-per-inch)) was captured at the same distance and was aligned using as reference points: the highest point of the Astragalus head, the extreme left point of the first distal phalanx and the extreme right point of the calcaneus.

\section{Results}

\subsection{About the model validation}

Results of the validation process can be seen in Table 3. The model generates a foot structure deformation as expected in a healthy patient, considering the foot anatomy from a lateral view under both a light loading (without soft tissue tension) and a normal standing load (soft tissue tension under normal conditions).

Table 3

Results of the validation process. The values correspond to the difference between the measured distance from each point to the ground, in two different loading conditions: Light loading and normal standing load.

\begin{tabular}{lll}
\hline Ref. point & Model prediction $(\mathrm{mm})$ & Patient average $(\mathrm{mm})$ \\
\hline AST & -0.33 & -0.33 (SD 0.14) \\
NAV & -0.27 & -0.26 (SD 0.04) \\
CUN & -0.26 & -0.19 (SD 0.08) \\
MTH1 & -0.07 & -0.08 (SD 0.03) \\
\hline
\end{tabular}

Additionally, the model generates a foot deformation that matches with normal clinical ranges: IMCB angle: $115^{\circ}$, Forefoot abduction (FA) angle $=17^{\circ}$ and the Kite's angle $=18^{\circ}$ (See Fig. 3 ( A-B)) (Younger et al., 2005; Haddad et al., 2011).

\subsection{The reference case}

The Normal simulation was used as a reference to be compared to each of the simulated case, while the Max. Deformation simulation was used to quantify the relative differences of each case. This difference comparison method is based on the experimental test reported by Tao et al. (2010). The results can be seen in Fig. 3 ( C-D).

\subsection{Foot bone structure deformation}

The first part of this study was focused on the evaluation of each tissue's ability to maintain both the foot bone structure and the plantar arch within normal ranges. We evaluated different conditions to quantify and compare the effect of the PF, SL and/or Tendons failure. In the All Tendons only case, the TPT, AT, PLT and the PBT were included, while the Passive Tissues case included the passive stabilizer tissues: PF, SL, SPL and LPL. The PF, the TPT and the SL were also tested in isolation. We used the Normal simulation as the base to measure the difference obtained in each case. The relative difference was measured using the Max. Deformation simulation shown in Section 3.2. All the results are shown in Table 4. We also included the AT as a measurement variable because we identify important differences in the tissue stress simulations as is shown below. The AT effect over these tissues has not been explicitly reported in the literature.

Table 4

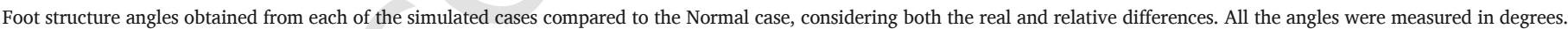
See Fig. 3 for measurements reference.

\begin{tabular}{|c|c|c|c|c|c|c|c|c|c|}
\hline \multirow[t]{2}{*}{ Simulation test } & \multicolumn{2}{|l|}{ Angles } & \multicolumn{3}{|c|}{ IMCB angle } & \multicolumn{2}{|c|}{ Kite's angle } & \multicolumn{2}{|c|}{ HA angle } \\
\hline & IMCB & Kite's & FA & Diff. & $(\%)$ & Diff. & (\%) & Diff. & (\%) \\
\hline Normal & 115 & 18 & 17 & 0 & 0 & 0 & 0 & 0 & 0 \\
\hline Max. Deformation & 130 & 23 & 26 & 15 & 100 & 5 & 100 & 9 & 100 \\
\hline Passive tissues & 116 & 20 & 15 & 1 & 7 & 2 & 40 & -2 & -22 \\
\hline All Tendons only & 122 & 18 & 21 & 7 & 47 & 0 & 0 & 4 & 44 \\
\hline TPT only & 121 & 22 & 24 & 6 & 40 & 4 & 80 & 7 & 78 \\
\hline PF only & 120 & 22 & 25 & 5 & 33 & 4 & 80 & 8 & 89 \\
\hline LS only & 122 & 20 & 18 & 7 & 47 & 2 & 40 & 1 & 11 \\
\hline $\mathrm{TPT}+\mathrm{PF}$ & 115 & 21 & 14 & 0 & 0 & 3 & 60 & -3 & -33 \\
\hline TPT + LS & 116 & 21 & 13 & 1 & 7 & 3 & 60 & -4 & -44 \\
\hline $\mathrm{TPT}+\mathrm{AT}$ & 125 & 18 & 22 & 10 & 67 & 0 & 0 & 5 & 56 \\
\hline $\mathrm{TPT}+\mathrm{PF}+\mathrm{AT}$ & 117 & 21 & 23 & 2 & 13 & 3 & 60 & 6 & 67 \\
\hline $\mathrm{TPT}+\mathrm{LS}+\mathrm{AT}$ & 121 & 22 & 17 & 6 & 40 & 4 & 80 & 0 & 0 \\
\hline
\end{tabular}




\subsection{Tibialis posterior tendon stress analysis}

The TPT is one of the main tissues related with AAFD. The second part of this study was oriented to measure the biomechanical stress (in $\mathrm{N} / \mathrm{m}^{2}$ ), generated in this tissue in different scenarios. The results were normalized from 0 to $60 \mathrm{~N} / \mathrm{m}^{2}$ in order to better show the differences, using as a reference the results obtained from the Normal simulation (Fig. 4A). The results are shown in Fig. 4. All the color scales show the highest value obtained from each of the simulated cases.

\subsection{Plantar fascia stress analysis}

As mentioned above, the plantar fascia is one of the main tissues associated with the plantar arch maintenance. We measured the biomechanical stress generated in this tissue in different scenarios. The re- sults are shown in Fig. 5. We normalized the results using as a reference the Normal simulation (Fig. 5A). Again, the highest values of all the simulated cases are shown to facilitate the comparison.

\subsection{Spring ligament stress analysis}

The SL is the other soft tissue that has been related in recent years with AAFD development. We quantify the biomechanical stress that is generated in this tissue in different scenarios using our model. The results obtained are shown in Fig. 6 . We normalized the results using the Normal simulation (about 0 to $45 \mathrm{~N} / \mathrm{m}^{2}$ ).

We do not present the biomechanical stress of the short plantar ligament nor the long plantar ligament in isolation, because their contribution to maintaining the plantar arch was not significant. The foot deformation results obtained from simulate in isolation each one of these tissues were close to the Max. Deformation simulation (consistent with

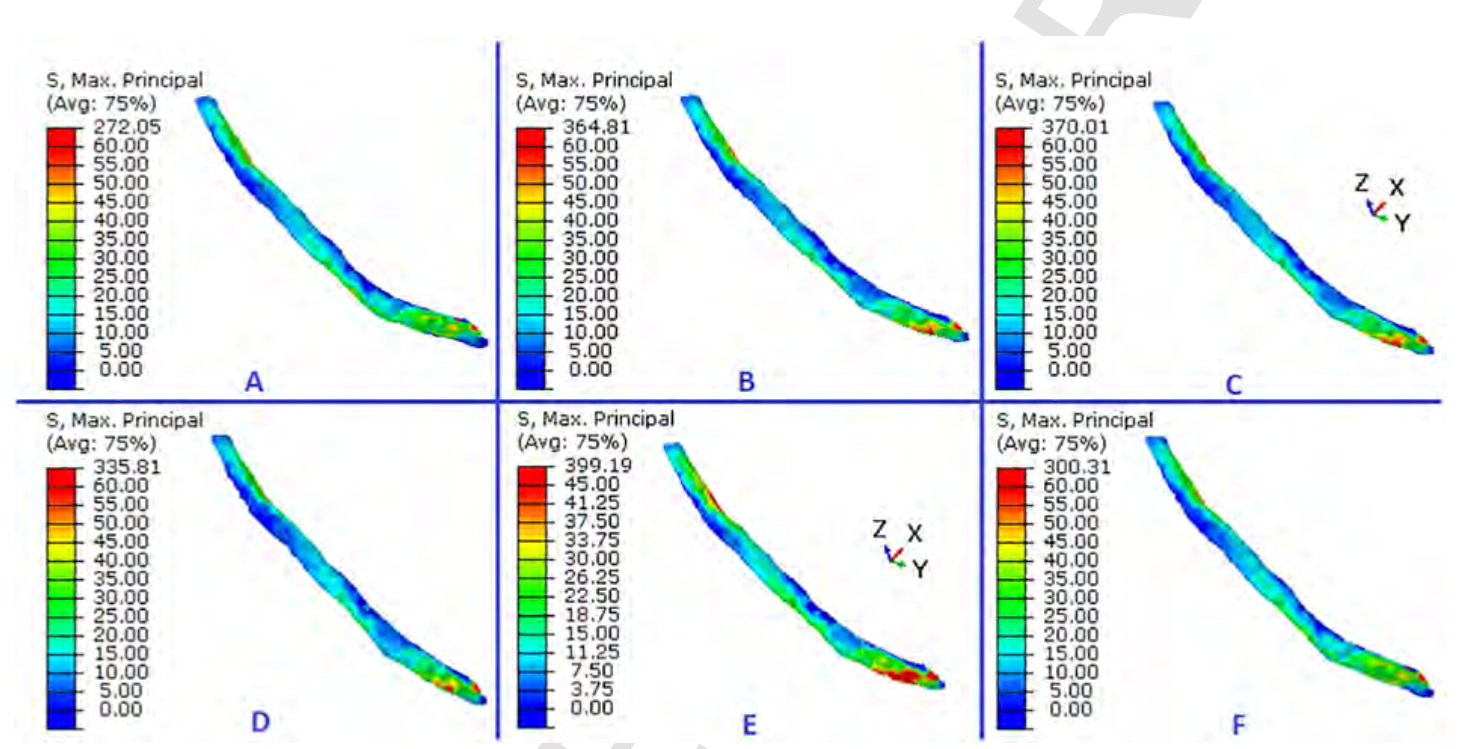

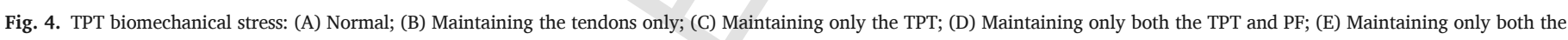
TPT and SL; (F) Maintaining only both the TPT and AT.

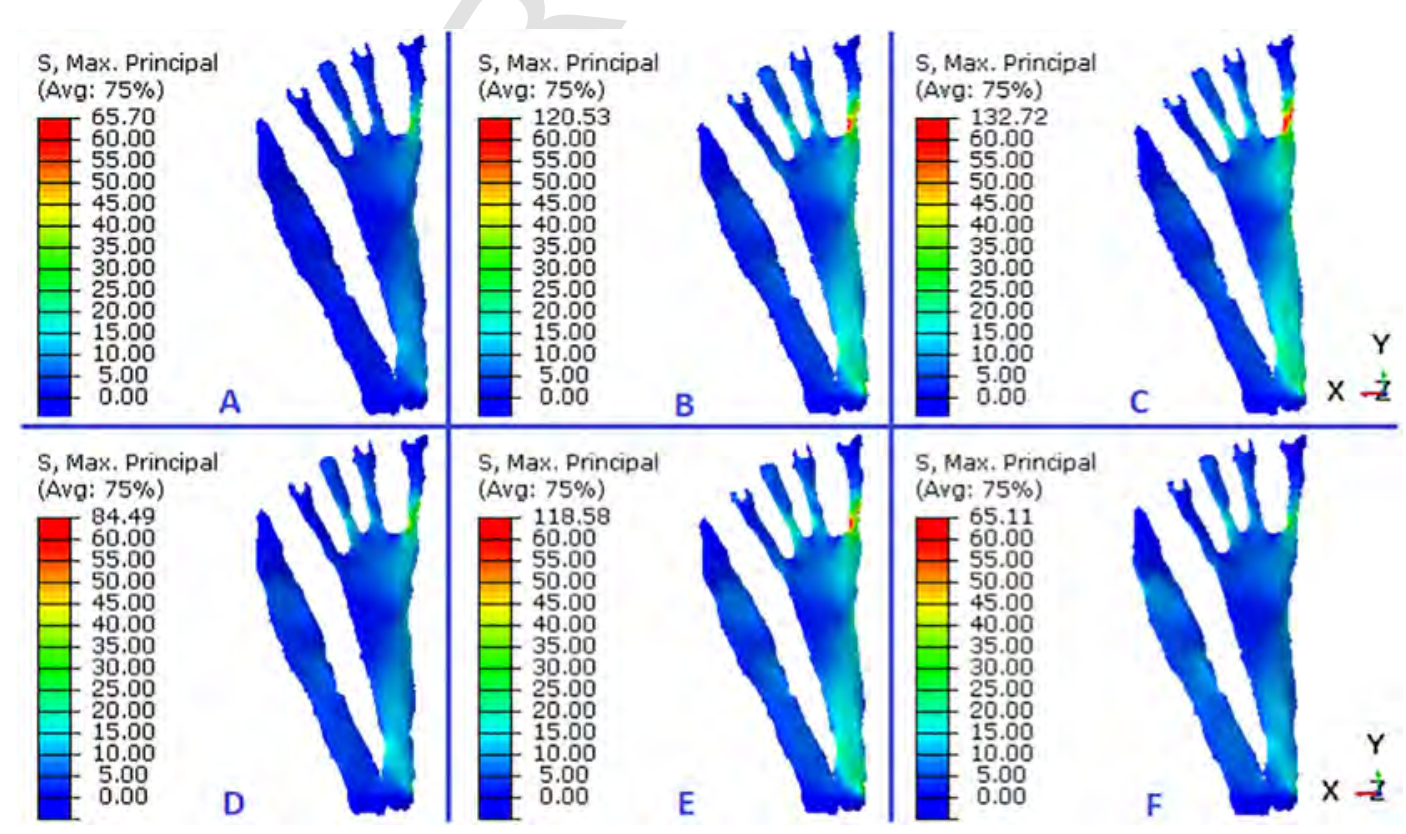

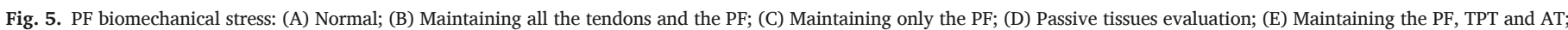
(F) Maintaining only both the PF and TPT. 


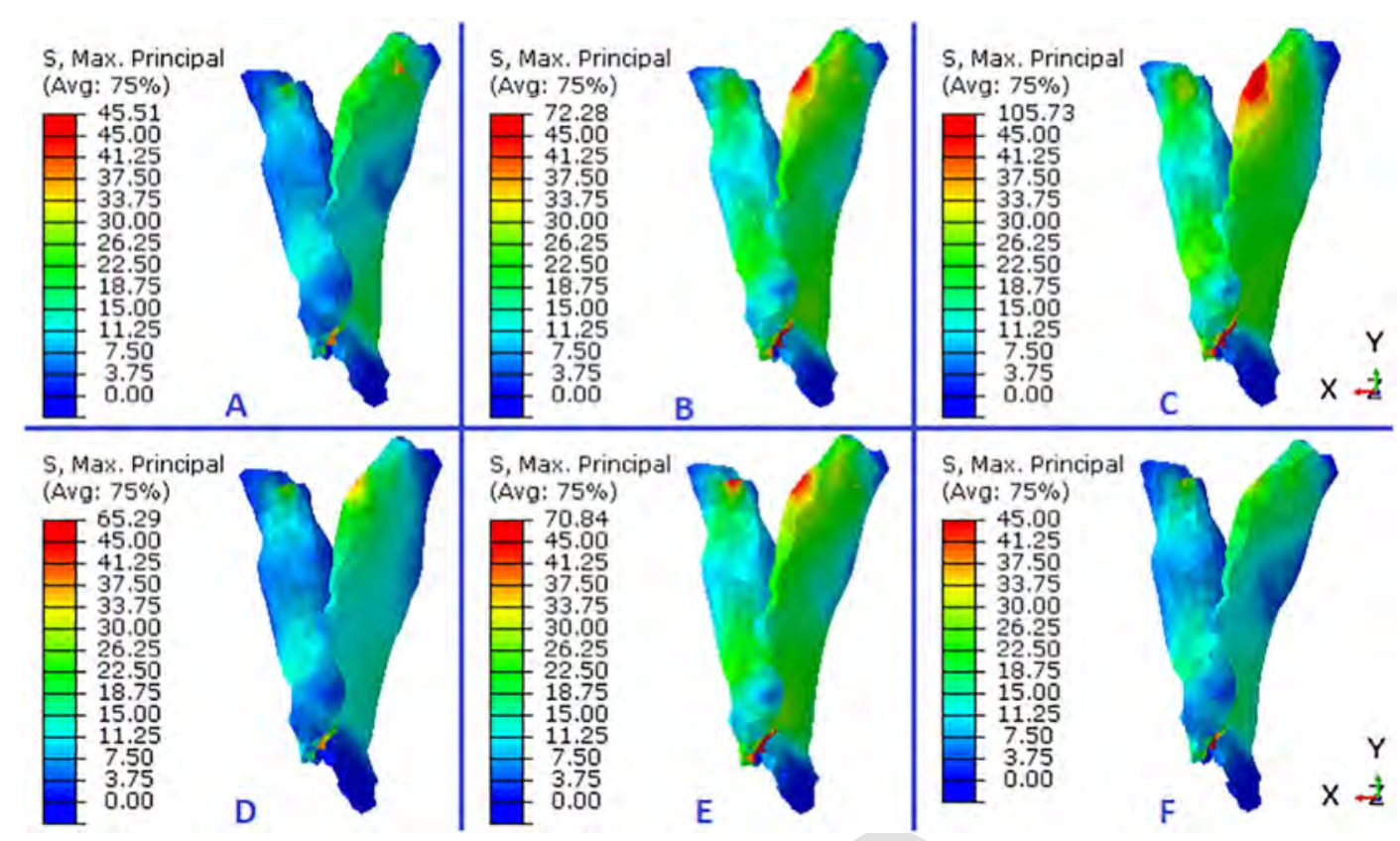

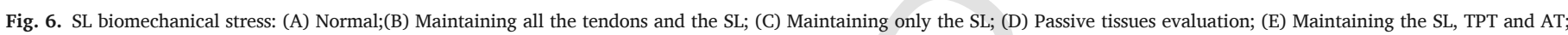
(F) Maintaining only both the SL and TPT.

the Huang et al. (1993) and Tao et al. (2010) experimental test results).

\section{Discussion}

Adult acquired flatfoot deformity is a debilitating condition with a wide range of treatment options. It is characterized by a painful flattening of the medial longitudinal arch. This pathology has been traditionally related to a tibialis posterior tendon deficiency (Kohls-Gatzoulis et al., 2004). However, some studies had found that the plantar calcaneonavicular ligament, also called the spring ligament, and the plantar fascia are also related to AAFD development (Deland, 2001; Tao et al., 2010; Toullec, 2015; Steginsky and Vora, 2017). There are many treatment options, but the choice depends on the physician's experience. Surgeons must decide between tendinous reinforcement techniques, corrective osteotomies or selective arthrodesis in the middle and hindfoot joints (Bluman et al., 2007; Vulcano et al., 2013; Smyth et al., 2017). This highly subjective process could be improved quantifying some biomechanical variables that have not to date been evaluated by experimental trials, such as biomechanical tissue stress.

This paper presents a computational human foot model that includes the main soft tissues traditionally related to AAFD development, evaluated following clinical criteria. Our model allowed us to evaluate the tibialis posterior tendon biomechanical stress under different conditions, as well as the stress generated in both the plantar fascia and the spring ligament, which are also related with the development of the disease. This evaluation, enabled us to calculate the contribution of each tissue in the plantar arch maintenance. The model reproduced the foot deformities observed in both normal and flatfoot cases. Its versatility also allowed us to evaluate in isolation the relative stress differences generated in the TPT, the PF and the SL, and in different combinations, including the analysis of the Achilles tendon's effect on all the plantar tissues stress.

Some authors have proposed computational models to evaluate the AAFD, including some tissue evaluations related to the maintenance of the plantar arch. Gefen (2002) and later Cheung and Zhang (2005) developed models that evaluate the biomechanical consequences of both a complete and partial PF removal. However, they simplified the bio- mechanical characteristics of the tissues, as well as their geometry, assuming the PF as a purely deformable element (bar elements). Wu (2007) proposed the first FE approach to evaluate the working of the plantar ligaments using a 2D model of the foot longitudinal arch. This model considered a detailed anatomical structure, as well as a nonlinear model for cartilages. Later, Tao et al. (2010) developed a detailed FE model, to evaluate the effect of the passive stabilizer tissues (FP, SL, SPL and LPL) on the plantar arch maintenance. In contrast to our proposal, these authors do not consider the hyperelastic behavior of the cartilage, the cortical and trabecular bone differentiation, nor the geometry suitable for the tissue stress study. Recently, some authors have considered complex computational models oriented to foot surgical planning (Spratley, 2013; Smith et al., 2016; Wang et al., 2016b; Wong et al., 2017). However, these proposals maintain the soft tissue simplifications which do not allow the analysis of the biomechanical stress, thus limiting the clinical interest of these proposals for AAFD studies.

The results of our study suggest that the PF is the main soft tissue that maintains the plantar arch within normal ranges, followed by both the SL and the TPT and, to a lesser extent, both the short and long plantar ligaments. These results are consistent with those of Huang et al. (1993) and Tao et al. (2010), who based their studies on experimental tests on cadaver models. Our model is capable of reproducing this type of study but also quantifying the biomechanical stress of each of the included tissues.

Although the biomechanical stress values generated cannot be assumed to represent the real stress values found in all people (because of inter-subject variability), we are able to quantify the relative differences generated in each one of the cases. Thus, there is a significant difference between the biomechanical stress generated in the tibialis posterior tendon compared to both the plantar fascia and the spring ligament. The order of magnitude generated in this tissue is almost four-times higher than the PF stress and six-times higher than the SL stress. However, the results show that the TPT cannot support the plantar arch by itself. The foot deformation obtained when simulating the model keeping only the TPT tissue, was similar to the Max. Deformation case. However, it did reduce the foot pronation, as the SL does. The simulation also showed that if the PF and the SL or TPT are in good 
condition, the plantar arch keeps within normal ranges, but if one of them fails, the entire foot structure will be affected.

The tension generated in both the PF and SL is reduced when the tendons are included. This means that the tendons help to support the foot loading as was expected. However, they cannot maintain the foot arch by themselves. Our findings also suggest that the Achilles Tendon increases considerably the biomechanical stress of all the plantar soft tissues. Figs. 4, 5 and 6, showed that the generated stress was higher when the Achilles tendon was included than when the tissues were simulated in isolation. These results can be explained considering that the action of the Achilles Tendon force vector pulls up the calcaneus head, generating an increase in the plantar arch aperture.

The foot deformation analysis shows that both the TPT and the SL action reduces the foot pronation. Additionally, the biomechanical stress generated in each one of the cases suggests that when one of these two tissues fails, the other one suffers considerable biomechanical stress increase (from about $300 \mathrm{~N} / \mathrm{m}^{2}$ to $370 \mathrm{~N} / \mathrm{m}^{2}$ in the TPT and from $45 \mathrm{~N} / \mathrm{m}^{2}$ to $105 \mathrm{~N} / \mathrm{m}^{2}$ in the SL). The results for both the Kite's angle and the FA angle were similar in simulations performed in isolation for each one of these tissues. The plantar arch aperture (measured by the IMCB angle) generated by simulating the model maintaining only the SL tissue was similar to that obtained with the TPT in isolation.

Furthermore, the model findings are consistent with the theory in which the tibialis posterior tendon has a secondary role, compared to both the PF and the SL in the plantar arch maintenance (elongation). Some authors consider that it is finally the tibialis posterior tendon which claudicates when the hindfoot has rotated around the tarsal-navicular joint. This explains the association between the TPT failure and the clinical signs of adult acquired flatfoot deformity (Richie, 2007).

The main limitation of our model is that the analyzed soft tissues were modeled as elastic-linear material. However, since the method applies a static calculation with small displacements and deformations, the error is insignificant. Finally, we consider that the model presented contributes towards increasing biomechanical knowledge of the action of foot tissues under loading tests and may be useful for the study of AAFD and related clinical research.

\section{Conclusions}

The biomechanical tissue stress measured with our model and the foot deformation generated, fit the theory in which the posterior tibial tendon has a secondary role in the plantar arch maintenance. This tendon cannot support the foot arch on its own, and its biomechanical stress increases considerably when other tissues fail. Our findings show that this tissue prevents the foot pronation as well as the spring ligament, while the plantar fascia is the main soft tissue that prevents foot elongation. These signs are the most relevant in AADF development. Thus, AADF treatments and evaluations should be focused mainly on these soft tissues. The model herein presented can be used to analyze different scenarios for AAFD clinical research.

\section{Author disclosure statement}

The authors declare that they have no competing financial interests.

\section{Acknowledgments}

The authors gratefully acknowledge the support of the Ministry of Economy and competitiveness MINECO of the Government of Spain through the project DPI2016-77016-R.

\section{References}

Arangio, G.A., Salathe, E.P., 2009. A biomechanical analysis of posterior tibial tendon dysfunction, medial displacement calcaneal osteotomy and flexor digitorum longus transfer in adult acquired flat foot. Clin. Biomech. 24, 385-390.

Bayod, J., Becerro-de Bengoa-Vallejo, R., Losa-Iglesias, M., Doblaré, M., 2012. Mechanical stress redistribution in the calcaneus after autologous bone harvesting. J. Biomech. 45, 1219-1226.

Bertani, A., Cappello, A., Benedetti, M., Simoncini, L., Catani, F., 1999. Flat foot functional evaluation using pattern recognition of ground reaction data. Clin. Biomech. 14, 484-493.

Bluman, E.M., Title, C.I., Myerson, M.S., 2007. Posterior tibial tendon rupture: a refined classification system. Foot Ankle Clin. 12, https://doi.org/10.1016/j.fcl.2007.03.003.

Burkhart, T.A., Andrews, D.M., Dunning, C.E., 2013. Finite element modeling mesh quality, energy balance and validation methods: a review with recommendations associated with the modeling of bone tissue. J. Biomech. 46, 1477-1488.

Cheung, J.T.-M., Zhang, M., 2005. A 3-dimensional finite element model of the human foot and ankle for insole design. Arch. Phys. Med. Rehabil. 86, 353-358.

Cheung, J.T.-M., Zhang, M., Leung, A.K.-L., Fan, Y.-B., 2005. Three-dimensional finite element analysis of the foot during standing-a material sensitivity study. J. Biomech. 38 , 1045-1054.

Deland, J.T., 2001. The adult acquired flatfoot and spring ligament complex: pathology and implications for treatment. Foot Ankle Clin. 6, 129-135.

Deschamps, K., Staes, F., Roosen, P., Nobels, F., Desloovere, K., Bruyninckx, H., Matricali, G.A., 2011. Body of evidence supporting the clinical use of 3d multisegment foot models: a systematic review. Gait Posture 33, 338-349.

Ergonautas, 2017. Ruler. Ergonautas and Universidad Politecnica de Valencia.

Fowble, V.A., Sands, A.K., 2004. Treatment of adult acquired pes plano abductovalgus (flatfoot deformity): procedures that preserve complex hindfoot motion. Operative Techniques in Orthopaedics 14 (1), 13-20.

Garcia-Aznar, J., Bayod, J., Rosas, A., Larrainzar, R., García-Bógalo, R., Doblaré, M., Llanos, L., 2009. Load transfer mechanism for different metatarsal geometries: a finite element study. J. Biomech. Eng. 131, 021011.

Gefen, A., 2002. Stress analysis of the standing foot following surgical plantar fascia release. J. Biomech. 35, 629-637.

Guha, A.R., Perera, A.M., 2012. Calcaneal osteotomy in the treatment of adult acquired flatfoot deformity. Foot Ankle Clin. 17, 247-258.

Haddad, S.L., Myerson, M.S., Younger, A., Anderson, R.B., Davis, W.H., Manoli, A., Davis, W.H., et al., 2011. Adult acquired flatfoot deformity. Foot Ankle Int. 32, 95-101.

Hidalgo, L.H., Arranz, J.C., Rodríguez, M.R., De La Pena, M.J., Alonso, R.C., Moreno, de Vega Fernández, V.M., 2014. Posterior tibial tendon dysfunction: what other struc tures are involved in the development of acquired adult flat foot?. Radiología 56 $247-256$.

Huang, C.-K., Kitaoka, H.B., An, K.-N., Chao, E.Y., 1993. Biomechanical evaluation of longitudinal arch stability. Foot Ankle 14, 353-357.

Kohls-Gatzoulis, J., Angel, J.C., Singh, D., Haddad, F., Livingstone, J., Berry, G., 2004. Tibialis posterior dysfunction: a common and treatable cause of adult acquired flatfoot. BMJ 329, 1328-1333. https://doi.org/10.1136/bmj.329.7478.1328.

Lee, M.S., Vanore, J.V., Thomas, J.L., Catanzariti, A.R., Kogler, G., Kravitz, S.R., Miller, S.J., Gassen, S.C., 2005. Diagnosis and treatment of adult flatfoot. J. Foot Ankle Surg. 44, 78-113.

Lladó, G.D., Net, R.B., Garcia, I.M., 2015. Double calcaneal osteotomy. Rev. Pie Tobillo 29, 101-107.

Mansour, J.M., 2003. Biomechanics of cartilage. Kinesiology 66-79.

Marchena, A., Cortés, M., Noguerón, G.G., 2013. Systematic review of flexible flatfoot treatments. Retrospective analysis (1977-2011). Rev. Int. Cienc. Podológicas 7, 9.

Morales Orcajo, E., E. Barbosa de las Casas, J. Bayod López, 2015. Computational Foot Modeling for Clinical Assessment. Ph.D. thesis Universidad de Zaragoza.

Pichler, W., Tesch, N.P., Grechenig, W., Tanzer, K., Grasslober, M., 2005. Anatomical variations of the flexor hallucis longus muscle and the consequences for tendon transfer. A cadaver study. Surg. Radiol. Anat. 27, 227-231.

Rabbito, M., Pohl, M.B., Humble, N., Ferber, R., 2011. Biomechanical and clinical factors related to stage I posterior tibial tendon dysfunction. J. Orthop. Sports Phys. Ther. 41, 776-784.

Richie, D.H., 2007. Biomechanics and clinical analysis of the adult acquired flatfoot. Clin. Podiatr. Med. Surg. 24, 617-644.

Shibuya, N., Jupiter, D.C., Ciliberti, L.J., VanBuren, V., La Fontaine, J., 2010. Characteristics of adult flatfoot in the United States. J. Foot Ankle Surg. 49, 363-368.

Smith, B.A., 2015. Computational Modeling to Assess Surgical Procedures for the Treat ment of Adult Acquired Flatfoot Deformity. Master's thesis Virginia Commonwealth University.

Smith, B.A., Adelaar, R.S., Wayne, J.S., 2016. Patient specific computational models to optimize surgical correction for flatfoot deformity. J. Orthop. Res.

Smyth, N.A., Aiyer, A.A., Kaplan, J.R., Carmody, C.A., Kadakia, A.R., 2017. Adult-acquired flatfoot deformity. Eur. J. Orthop. Surg. Traumatol. 27, 433-439.

Song, J., Hillstrom, H., Secord, D., Levitt, J., 1996. Foot type biomechanics. Comparison of planus and rectus foot types. J. Am. Podiatr. Med. Assoc. 86, 16-23.

Spratley, E.M., 2013. Patient-Specific Modeling Of Adult Acquired Flatfoot Deformity Before And After Surgery. Dissertation Virginia Commonwealth University.

Steginsky, B., Vora, A., 2017. What to do with the spring ligament. Foot Ankle Clin. 
Tao, K., Ji, W.-T., Wang, D.-M., Wang, C.-T., Wang, X., 2010. Relative contributions of plantar fascia and ligaments on the arch static stability: a finite element study. Biomed. Tech. Biomed. Eng. 55, 265-271.

Tao, K., Wang, D., Wang, C., Wang, X., Liu, A., Nester, C.J., Howard, D., 2009. An in vivo experimental validation of a computational model of human foot. J. Bionic Eng. 6, 387-397.

Toullec, E., 2015. Adult flatfoot. Orthop. Traumatol. Surg. Res. 101, S11-S17.

Valladar, M.T.M., Carsi, V.V., Carrera, J.M.G., Adrien, X.G., Paños, J.P.G., Rodríguez, J.E.G., 2015. Commentary double calcaneal osteotomy. Rev. Pie Tobillo 29, 107-108.

Viceconti, M., Olsen, S., Nolte, L.-P., Burton, K., 2005. Extracting clinically relevant data from finite element simulations. Clin. Biomech. 20, 451-454.

Vulcano, E., Deland, J.T., Ellis, S.J., 2013. Approach and treatment of the adult acquired flatfoot deformity. Curr. Rev. Muscoskelet. Med. 6, 294-303.

Wang, Y., Wong, D.W.-C., Zhang, M., 2016. Computational models of the foot and ankle for pathomechanics and clinical applications: a review. Ann. Biomed. Eng. 44, 213-221.
Wang, Z., Imai, K., Kido, M., Ikoma, K., Hirai, S., 2015. Fe model of a flatfoot deformity for improving surgical planning. In: SIMULIA Community Conference.

Wang, Z., Imai, K., Kido, M., Ikoma, K., Hirai, S., 2016. Study of surgical simulation of flatfoot using a finite element model. In: Innovation in Medicine and Healthcare 2015, Springer, pp. 353-363.

Wong, D.W.-C., Wang, Y., Leung, A.K.-L., Yang, M., Zhang, M., 2017. Finite element simulation on posterior tibial tendinopathy: load transfer alteration and implications to the onset of pes planus. Clin. Biomech. https://doi.org/10.1016/j.clinbiomech.2017. 11.001.

Wright, D., Rennels, D., 1964. A study of the elastic properties of plantar fascia. JBJS 46, $482-492$.

Wu, L., 2007. Nonlinear finite element analysis for musculoskeletal biomechanics of medial and lateral plantar longitudinal arch of virtual Chinese human after plantar ligamentous structure failures. Clin. Biomech. 22, 221-229.

Younger, A.S., Sawatzky, B., Dryden, P., 2005. Radiographic assessment of adult flatfoot. Foot Ankle Int. 26, 820-825. 